BULL. AUSTRAL. MATH. SOC.

VOL. 34 (1986) 461-472

\title{
A NOTE ON N-HARMONIC MAJORANTS
}

\author{
Hong Oh Kim and Chang Ock Lee
}

Suppose $D(v)$ is the Dirichlet integral of a function $v$ defined on the unit disc $U$ in the complex plane. It is well known that if $v$ is a harmonic function in $U$ with $D(v)<\infty$, then for each $p, 0<p<\infty,|v|^{p}$ has a harmonic majorant in $U$.

We define the "iterated" Dirichlet integral $D_{n}(v)$ for a function $v$ on the polydisc $u^{n}$ of $c^{n}$ and prove the polydisc version of the well known fact above:

If $v$ is an $n$-harmonic function in $U^{n}$ with $D_{n}(v)<\infty$, then for each $p, 0<p<\infty,|v| p$ has an $n$-harmonic majorant in $U^{n}$.

\section{Introduction}

For a differentiable real function $v$ defined in the polydisc,

$$
U^{n}=\left\{\left(z_{1}, \ldots z_{n}\right):\left|z_{i}\right|<1 \text { for } 1 \leq j \leq n\right\}
$$

in $C^{n}$, we define the "iterated" Dirichlet integral $D_{n}(v)$ as

$$
\int u_{u}^{n}\left|\nabla_{1} \otimes \ldots \otimes \nabla_{n} v\right|^{2} d x d y .
$$

where $z_{j}=x_{j}+i y_{j}, d x=d x_{1} \ldots d x_{n}, d y=d y_{1} \ldots d y_{n}$ and

$$
\nabla_{1} \otimes \ldots \otimes \nabla_{n}=\left(\frac{\partial}{\partial x_{1}}, \frac{\partial}{\partial y_{1}}\right) \otimes \ldots \otimes\left(\frac{\partial}{\partial x_{n}}, \frac{\partial}{\partial y_{n}}\right)
$$

Received 6 February 1986.

Copyright Clearance Centre, Inc. Serial-fee code: 0004-9727/86 $\$ A 2.00+0.00$. 
For example, we have

$$
\left|\nabla_{1} \otimes \nabla_{2} v\right|^{2}=\left|\frac{\partial^{2} v}{\partial x_{1} \partial x_{2}}\right|^{2}+\left|\frac{\partial^{2} y}{\partial x_{1} \partial y_{2}}\right|^{2}+\left|\frac{\partial^{2} v}{\partial y_{1} \partial x_{2}}\right|^{2}+\left|\frac{\partial^{2} v}{\partial y_{1} \partial y_{2}}\right|^{2} .
$$

If $n=1, D_{1}(v)$ is the usual Dirichlet integral of $v$.

A continuous function $v$ is an open set in $c^{n}$ is $n$-harmonic if $v$ is harmonic in each complex variable separately, that is,

$$
\frac{\partial^{2} v}{\partial x_{j}^{2}}+\frac{\partial^{2} v}{\partial y_{j}^{2}}=0,(1 \leq j \leq n) \text {. }
$$

The function $v$ on $U^{n}$ has an $n$-harmonic majorant if there is an $n$-harmonic function $V$ such that $v(z) \leq V(z)$ throughout $U^{n}$.

Let $h^{p}\left(u^{n}\right), 0<p<\infty$, be the class of all $n$-harmonic functions $v$ in $U^{n}$ for which

where

$$
\|v\|_{p}=\sup _{0 \leq r_{1}, \ldots, r_{n}<1} M_{p}\left(r_{1}, \ldots, r_{n} ; v\right)<\infty
$$

$$
M_{p}\left(r_{1}, \ldots, r_{n} ; v\right)=\left(\int_{0}^{2 \pi} \ldots \int_{0}^{2 \pi} \mid v\left(r_{1} e^{i \theta_{1}}, \ldots, r_{n} e^{i \theta_{n}} p^{\frac{d \theta_{1}}{2 \pi}} \ldots \frac{d \theta_{n}}{2 \pi}\right) 1 / p\right.
$$

For $p \geq 1, M_{p}$ is an increasing function of $r_{1}, \ldots, r_{n}$,

separately. If $v \in h^{p}\left(U^{n}\right), p \geq 1$, it is known that the radial limit

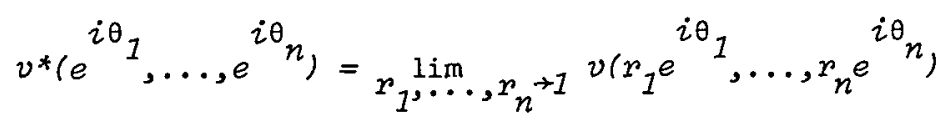

of $v$ exists almost everywhere and it is an $L^{p}$-function on the distinguished boundary.

$$
T^{n}=\left\{\left(z_{1}, \ldots, z_{n}\right):\left|z_{j}\right|=1 \text { for } 1 \leq j \leq n\right\} \text {. }
$$

Moreover, $\|v\|_{p}=\left\|v^{*}\right\|_{L^{p}\left(T^{n}\right)}$. Also, if $v \in h^{p}\left(U^{n}\right), p>1$, then $v$ is equal to the iterated Poisson integral of its radial limit function $v^{*}$. That is,

$v\left(z_{1}, \ldots, z_{n}\right)=\int \ldots_{T^{n}} \int P_{r_{1}}\left(\theta_{1}-t_{1}\right) \ldots P_{r_{n}}\left(\theta_{n}-t_{n}\right) v^{*}\left(e^{i t_{1}}, \ldots, e^{i t_{n}}\right) \frac{d t_{1}}{2 \pi} \ldots \frac{d t_{n}}{2 \pi}$ 
where $P_{p}(\theta-t)$ is the Poisson kernel for $U$. See $[1,3,8]$ for more about $h^{p}$.

The prototype of our main theme is the following well-known theorem (see [9] for example):

Let $v$ be a harmonic function in $U$ with $D_{1}(v)<\infty$. Then for each $p, 0<p<\infty$, the function $|v|^{p}$ has a harmonic majorant in $U$.

We prove the polydisc version of the theorem above:

MAIN THEOREM. Let $v$ be an n-harmonic function in $U^{n}$ with $D_{n}(v)<\infty$. Then for each $p, 0<p<\infty$, the function $|v| p$ has an $n$-harmonic majorant in $U^{n}$.

We consider only the case $n=2$, but the procedure can be repeated for an arbitrary $n$.

\section{The iterated Dirichlet integral}

PROPOSITION 2.1. If $v$ is a 2-harmonic function in $U^{2}$, then

$$
D_{2}(v)=4 \quad \iint_{U^{2}}\left|\frac{\partial^{2} v}{\partial r_{1} \partial r_{2}}\right|^{2} d x d y
$$

Proof. By an elementary calculation, we see that $\left|\nabla_{1} \otimes \nabla_{2} v\right|^{2}$

$$
\begin{aligned}
& =\left|\frac{\partial^{2} v}{\partial x_{1} \partial x_{2}}\right|^{2}+\left|\frac{\partial^{2} v}{\partial x_{1} \partial y_{2}}\right|^{2}+\left|\frac{\partial^{2} v}{\partial y_{1} \partial x_{2}}\right|^{2}+\left|\frac{\partial^{2} v}{\partial y_{1} \partial y_{2}}\right|^{2} . \\
& =\left|\frac{\partial^{2} v}{\partial r_{1} \partial r_{2}}\right|^{2}+\frac{1}{r_{1}{ }^{2}}\left|\frac{\partial^{2} v}{\partial \theta_{1} \partial r_{2}}\right|^{2}+\frac{1}{r_{2}{ }^{2}}\left|\frac{\partial^{2} v}{\partial r_{1} \partial \theta_{2}}\right|^{2}+\frac{1}{r_{1}{ }^{2} r_{2}{ }^{2}}\left|\frac{\partial^{2} v}{\partial \theta_{1} \partial \theta_{2}}\right|^{2} .
\end{aligned}
$$

Since $v$ can be expanded as

$$
v\left(r_{1} e^{i \theta}, r_{2} e^{i \theta_{2}}\right)=\sum_{k \in Z^{2}} i(k) r_{1}{ }_{r_{2}}^{\left|k_{1}\right|}\left|k_{2}\right| e^{i k_{1} \theta_{1}+i k_{2} \theta_{2}}
$$

where $k=\left(k_{1}, k_{2}\right)$, a pair of integers, and $\hat{v}(k)$ is the multiple Fourier coefficient of $v$, we have, by Parseval's identity, 
$\int_{0}^{2 \pi} \int_{0}^{2 \pi}\left|\frac{\partial^{2} v}{\partial r_{1} \partial r_{2}}\right|^{2} d \theta_{1} d \theta_{2}=(2 \pi)^{2} \sum_{k \in Z} 2\left|k_{1}\right|^{2}\left|k_{2}\right|^{2}|\hat{v}(k)|^{2} r_{1}{ }^{2\left|k_{1}\right|-2} 2\left|k_{2}\right|-2$, $\int_{0}^{2 \pi} \int_{0}^{2 \pi}\left|\frac{\partial^{2} v}{\partial \theta_{1} \partial r_{2}}\right|^{2} d \theta_{1} d \theta_{2}=(2 \pi)^{2} \sum_{k \in Z^{2}}\left|k_{1}\right|^{2}\left|k_{2}\right|^{2}|\hat{v}(k)|^{2} r_{1}{ }^{2\left|k_{1}\right|}{ }_{r_{2}} 2\left|k_{2}\right|-2$, $\int_{0}^{2 \pi} \int_{0}^{2 \pi}\left|\frac{\partial^{2} v}{\partial r_{1}{ }^{\partial \theta_{2}}}\right|^{2} d \theta_{1} d \theta_{2}=(2 \pi)^{2} \sum_{k \in Z^{2}}\left|k_{1}\right|^{2}\left|k_{2}\right|^{2}|\hat{v}(k)|^{2} r_{1}^{2\left|k_{1}\right|-2}{ }_{r_{2}}^{2\left|k_{2}\right|}$, and $\int_{0}^{2 \pi} \int_{0}^{2 \pi}\left|\frac{\partial^{2} v}{\partial \theta_{1} \partial \theta_{2}}\right|^{2} d \theta_{1} d \theta_{2}=(2 \pi)^{2} \sum_{k \in Z^{2}}\left|k_{1}\right|^{2}\left|k_{2}\right|^{2}|\hat{v}(k)|^{2} r_{1}{ }^{2\left|k_{1}\right|} r_{2} 2\left|k_{2}\right|$. So $\int \underset{U^{2}}{. .}\left|\frac{\partial^{2} v}{\partial r_{1} \partial r_{2}}\right|^{2} d x d y=\int \frac{U_{U^{2}}}{r_{1}{ }^{2}}\left|\frac{\partial^{2} v}{\partial \theta_{1} \partial r_{2}}\right|^{2} d x d y$

$$
\begin{aligned}
& =\int \ddot{U^{2}} \int \frac{1}{r_{2}{ }^{2}}\left|\frac{\partial^{2} v}{\partial r_{1}{ }^{\partial \theta_{2}}}\right|^{2} d x d y \\
& =\int \ddot{U^{2}} \int \frac{1}{r_{1}{ }^{2} r_{2}{ }^{2}}\left|\frac{\partial^{2} v}{\partial \theta_{1}{ }^{\partial \theta_{2}}}\right|^{2} d x d y \\
& =\pi^{2} \sum_{k \in Z^{2}}\left|k_{1}\right|\left|k_{2}\right||\hat{v}(k)|^{2} .
\end{aligned}
$$

Hence we have

$$
D_{2}(v)=4 \int \ddot{U^{2}} \int\left|\frac{\partial^{2} v}{\partial r_{1} \partial r_{2}}\right|^{2} d x d y
$$

In general, for an $n$-harmonic function $v$ in $U^{n}$, we have

$$
D_{n}(v)=2^{n} \int_{U^{n}} \ldots \int\left|\frac{\partial^{n} v}{\partial r_{1} \cdot \partial_{n} r^{2}}\right|^{2} d x d y .
$$

Let $\alpha$ be a real number and let

$$
v\left(z_{1}, z_{2}\right)=\sum_{k \in Z^{2}} \hat{v}(k) r_{1}\left|k_{1}\right| r_{2}\left|k_{2}\right| e^{i k_{1} \theta_{1}+i k_{2} \theta_{2}}
$$

be a 2-harmonic function on $U^{2}$. The fractional derivative of $v$ of 
order $\alpha$ is defined as

$D^{\alpha, \alpha} v_{\left(z_{1}, z_{2}\right)}=\sum_{k \in Z^{2}}\left(1+\left|k_{1}\right|^{\alpha}\left(1+\left|k_{2}\right|\right)^{\alpha} \hat{v}(k) r_{1}{ }_{r_{2}}^{\left|k_{1}\right|}\left|k_{2}\right| \sum^{i k_{1} \theta_{1}+i k_{2} \theta_{2}}\right.$.

The fractional integral of $v$ of order $\alpha$ is defined as $I^{\alpha, \alpha} v=D^{-\alpha,-\alpha} v$. If $\alpha>0$, the following integral representation can easily be verified:

$$
I^{\alpha, \alpha} v\left(z_{1}, z_{2}\right)=\frac{1}{\Gamma(\alpha)^{2}} \int_{0}^{1} \int_{0}^{1}\left(\log \frac{1}{\rho}\right)^{\alpha-1}\left(\log \frac{1}{\sigma}\right)^{\alpha-1} v\left(\rho z_{1}, \sigma z_{2}\right) d \rho d \sigma .
$$

PROPOSITION 2.2. Let $v$ be a 2-harmonic function in $u^{2}$. Then. $D_{2}(v)<\infty$ if and only if $D^{\frac{1}{2}, \frac{1}{2}} v \in h^{2}\left(U^{2}\right)$.

Proof. By Parseval's identity we have

$$
|| D^{\frac{1}{2}, \frac{1}{2}} v||_{2}^{2}=\sum_{k \in Z^{2}}\left(1+\left|k_{1}\right|\right)\left(1+\left|k_{2}\right|\right)|\hat{v}(k)|^{2}
$$

By (2.1), we obtain that $D^{\frac{1}{2}, \frac{1}{2}} v \in h^{2}\left(U^{2}\right)$ if and only if

$$
\int \ddot{U} \int\left|\frac{\partial^{2} v}{\partial r_{1} \partial r_{2}}\right|^{2} d x d y<\infty \text {, }
$$

or, equivalently, if and only if $D_{2}(v)<\infty$.

Generally, for an $n$-harmonic function $v$ in $U^{n}, D_{n}(v)<\infty$

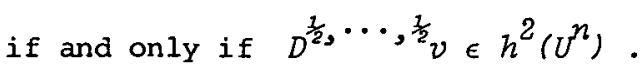

The following proposition is known, but we include a proof.

PROPOSITION 2.3. For $p>1, v \in h^{p}\left(U^{2}\right)$ if and only if $|v|^{p}$ has a 2-harmonic majorant.

Proof. Let $v$ be a 2-harmonic majorant of $|v|^{p}$. Then we have, by the mean value property,

$$
\begin{aligned}
\int_{0}^{2 \pi} \int_{0}^{2 \pi}\left|v\left(r_{1} e^{i \theta_{1}}, r_{2} e^{i \theta_{2}}\right)\right|^{p} \frac{d \theta_{1}}{2 \pi} \frac{d \theta_{2}}{2 \pi} & \leq \int_{0}^{2 \pi} \int_{0}^{2 \pi} V\left(r_{1} e^{i \theta_{1}}, r_{2} e^{i \theta_{2}}\right) \frac{d \theta_{1}}{2 \pi} \frac{d \theta_{2}}{2 \pi} \\
& =V(0,0)<\infty ;
\end{aligned}
$$

so $v \in h^{p}\left(v^{2}\right)$. 
If $v \in h^{p}\left(\nu^{2}\right)$ for $p>1$, we have

$$
v\left(z_{1}, z_{2}\right)=\int_{0}^{2 \pi} \int_{0}^{2 \pi} P_{r_{1}}\left(\theta_{1}-t_{1}\right) P_{r_{2}}\left(\theta_{2}-t_{2}\right) v^{*}\left(e^{i t_{1}}, e^{i t_{2}}\right) \frac{d t_{1}}{2 \pi} \frac{d t_{2}}{2 \pi},
$$

where $P_{r}(\theta-t)$ is Poisson kernel for $U$. We apply Jensen's inequality to get

(2.2) $\left|v\left(z_{1}, z_{2}\right)\right|^{p} \leq \int_{0}^{2 \pi} \int_{0}^{2 \pi} P_{r_{1}}\left(\theta_{1}-t_{1}\right) P_{r_{2}}\left(\theta_{2}-t_{2}\right)\left|v^{*}\left(e^{i t_{1}}, e^{i t_{2}}\right)\right|^{p} \frac{d t_{1}}{2 \pi} \frac{d t_{2}}{2 \pi}$.

But the right hand side of (2.2) is a 2-harmonic function and so a 2-harmonic majorant of $|v|^{p}$. This completes the proof.

\section{A theorem of Hardy and Littlewood}

If $v$ is a function in $U^{2}, v(\cdot, w)$ denotes the function $z \rightarrow v(z, w)$ with $w$ fixed and $v_{r, s}$ the function $(z, w) \rightarrow v(r z, s w)$. Throughout this paper, $C(\ldots)$ denotes a positive constant depending only on the argument (...) and it may vary from occurence to occurence even in the proof of the same theorem.

LEMMA 3.1. [1] For $\alpha>1$

$$
\int_{0}^{2 \pi}\left|1-r e^{i \theta}\right|^{-\alpha} d e=0(1-r)^{-\alpha+1},(r \rightarrow 1) \text {. }
$$

LEMMA 3.2. If $v \in h^{p}\left(U^{2}\right), p>1$, then

and

$$
|v(z, w)| \leq C(p)|| v(z, \cdot) \|_{p}(1-|w|)^{-1 / p}
$$

$$
|v(z, w)| \leq\left. C(p)|| v(\cdot, w)\right|_{p}(1-|z|)^{-1 / p} .
$$

Proof. For a fixed $z$, we have

$$
v(z, x)=\int_{0}^{2 \pi} P_{s}(n-t) v^{*}\left(z, e^{i t}\right) \frac{d t}{2 \pi}
$$

where $w=s e^{i n}$ and $v^{*}\left(z, e^{i t}\right)=\lim _{\delta \rightarrow 1} v^{*}\left(z, s e^{i t}\right)$. Using Hölder's inequality, we have 


$$
\begin{aligned}
|v(z, w)| & \leq\left(\int_{0}^{2 \pi}\left|v^{*}\left(z, e^{i t}\right)\right|^{p} \frac{d t}{2 \pi}\right)^{1 / p}\left(\int_{0}^{2 \pi}\left(\frac{1-|w|^{2}}{\left|e^{i t}-w\right|^{2}}\right)^{q} \frac{d t}{2 \pi}\right)^{1 / q} \\
& \leq\|v(z, \cdot)\|_{p}\left(1-|w|^{2}\right)\left(\int_{0}^{2 \pi} \frac{1}{\left|e^{i t}-s e^{i n}\right|^{2 q}} \frac{d t}{2 \pi}\right)^{1 / q},
\end{aligned}
$$

where $q$ is the conjugate index of $p$. By Lemma 3.1 there exists a constant $C(p)$ such that

$$
\int_{0}^{2 \pi} \frac{1}{\left|e^{i t}-8 e^{i n}\right|^{2 q}} \frac{d t}{2 \pi} \leq C(p)(1-|w|)^{1-2 q} .
$$

Hence

$$
|v(z, w)| \leq C(p)|| v(z, \cdot)||_{p}(1-|w|)^{-1 / p}
$$

LEMMA 3.3. If $v \in h^{p}\left(U^{2}\right), p>1$, then

$$
\|v(\cdot, w)\|_{p} \leq C(p) \| v||_{p}(1-|w|)^{-1 / p} \text {. }
$$

Proof. We use Lemma 3.2 and the monotone convergence theorem (MCT) to get

$$
\begin{aligned}
& \| v(\cdot, w)||_{p}^{p}=\lim _{r \rightarrow 1} \int_{0}^{2 \pi}\left|v\left(r e^{i \theta}, w\right)\right|^{p} \frac{d \theta}{2 \pi} \\
& \leq C(p)(1-|w|)^{-1} \lim _{r \rightarrow 1} \int_{0}^{2 \pi}|| v\left(r e^{i \theta}, \cdot\right)||_{p}^{p} \frac{d \theta}{2 \pi} \quad \text { (Lemma 3.2) } \\
& =c(p)(1-|w|)^{-1} \lim _{r \rightarrow 1} \int_{0}^{2 \pi} \lim _{s \rightarrow 1} \int_{0}^{2 \pi}\left|v\left(r e^{i \theta}, s e^{i n}\right)\right| p \frac{d n}{2 \pi} \frac{d \theta}{2 \pi} \\
& =C(p)(1-|\omega|)^{-1} \lim _{\substack{r \rightarrow 1 \\
s \rightarrow 1}} \int_{0}^{2 \pi} \int_{0}^{2 \pi} \mid v\left(r e^{i \theta},\left.s e^{i n}\right|^{p} \frac{d n}{2 \pi} \frac{d \theta}{2 \pi} \quad\right. \text { (MCT) } \\
& =C(p)(1-|w|)^{-1}|| v||_{p}^{p} .
\end{aligned}
$$

Hence we have

$$
\|v(\cdot, w)\|_{p} \leq c(p) \| v||_{p}(1-|w|)^{-1 / p}
$$


LEMMA 3.4. If $v \in h^{p}\left(U^{2}\right), p>1$, and if

$$
M(n)=\sup _{0 \leq \sigma<1}\left(\int_{0}^{2 \pi}\left|v\left(r e^{i \theta}, \sigma s e^{i n}\right)\right|^{p} \frac{d \theta}{2 \pi}\right)^{1 / p},
$$

then

$$
\int_{0}^{2 \pi} M(\eta)^{p} \frac{d \eta}{2 \pi} \leq C(p) M_{p}(r, s ; v)^{p} \text {. }
$$

Proof. We use Fubini's theorem and apply the Hardy-Littlewood maximal theorem ('Max") [1, p.11] to the harmonic function $v(z, \cdot)$ to get

$$
\begin{aligned}
\int_{0}^{2 \pi} M(n)^{p} \frac{d n}{2 \pi} & =\int_{0}^{2 \pi} \sup _{0 \leq \sigma<1} \int_{0}^{2 \pi}\left|v\left(r e^{i \theta}, \sigma s e^{i n}\right)\right|^{p} \frac{d \theta}{2 \pi} \frac{d \eta}{2 \pi} \\
& \leq \int_{0}^{2 \pi} \int_{0}^{2 \pi} \sup _{0 \leq \sigma<1}\left|v\left(r e^{i \theta}, \sigma s e^{i n}\right)\right|^{p} \frac{d \theta}{2 \pi} \frac{d n}{2 \pi} \\
& =\int_{0}^{2 \pi}\left(\int_{0}^{2 \pi} \sup _{0 \leq \sigma<1}\left|v\left(r e^{i \theta}, \sigma s e^{i n}\right)\right|^{p} \frac{d \eta}{2 \pi}\right) \frac{d \theta}{2 \pi} \text { (Fubini) } \\
& \leq C(p) \int_{0}^{2 \pi} \int_{0}^{2 \pi}\left|v\left(r e^{i \theta}, s e^{i n}\right)\right|^{p} \frac{d n}{2 \pi} \frac{d \theta}{2 \pi} \quad \text { ('Max') } \\
& =C(p) M_{p}(r, s ; v)^{p} .
\end{aligned}
$$

Now, we can proceed as in the proof of Theorem 2.2 in [7] to prove the following theorem. We give its proof for the sake of completeness. The corresponding theorem on holomorphic functions on the unit disc was proved by Hardy and Littlewood $[4,5]$ and by Flett [2], and on the polydisc $U^{n}$, by Kim [7].

THEOREM 3.5. If $0<\alpha<\frac{1}{p}$ and if $v \in h^{p}\left(U^{2}\right), p>1$, then $I^{\alpha, \alpha} v \in h^{q}\left(v^{2}\right)$ where $q=\frac{p}{1-\alpha p}$

Proof. Set

$$
M\left(r e^{i \theta}, \sigma w\right)=\sup _{0 \leq \rho<1}\left|v\left(\rho r e^{i \theta}, \sigma \omega\right)\right| .
$$




\section{n-Harmonic Majorants}

We write $z=r e^{i \theta}$ and $w=s e^{i \eta}$. By Lemma 3.2

$$
|v(\rho z, \sigma w)| \leq C(p)|| v_{r, s}\left(\cdot, \sigma e^{i \eta}\right)||_{p}(1-\rho)^{-1 / p} .
$$

By (3.1) and (3.2), we have

$$
\begin{aligned}
& \int_{0}^{1}(1-\rho)^{\alpha-1}\left|v\left(\rho r e^{i \theta}, \sigma s e^{i n}\right)\right| d \rho \\
& \leq C(p)|| v_{r, s}\left(\cdot, \sigma e^{i n}\right)||_{p} \int_{0}^{\lambda}(1-\rho)^{\alpha-\frac{1}{p}-1} d \rho \\
& +M\left(r e^{i \theta}, \sigma w\right) \int_{\lambda}^{1}(1-\rho)^{\alpha-1} d \rho .
\end{aligned}
$$

If $\left\|v_{r, 8}\left(\cdot, \sigma e^{i n}\right)\right\|_{p} \geq M\left(r e^{i \theta}, \sigma \omega\right)$, we set $\lambda=0$ in (3.4). (3.3) is then dominated by $C(\alpha)\left\|v_{r, s}\left(\cdot, \sigma e^{i \eta}\right)\right\|_{p}$. If $\left\|v_{r, s}\left(\cdot, \sigma e^{i n}\right)\right\|_{p}$ $<M\left(r e^{i \theta}, o w\right)$, we set

$$
\lambda=1-\left(\frac{\left\|v_{r, \varepsilon}\left(\cdot, \sigma e^{i n}\right)\right\|_{p}}{M\left(r e^{i \theta}, \sigma w\right)}\right)^{p} .
$$

(3.3) is then dominated by

$$
C(\alpha, p)\left\|v_{r, s}\left(\cdot, \sigma e^{i \eta}\right)\right\|_{p}^{\alpha p} M\left(r e^{i \theta}, \sigma \omega\right)^{1-\alpha p} .
$$

Hence for any $n$, we have

$$
\text { (3.5) } \begin{aligned}
& \int_{0}^{1}(1-\rho)^{\alpha-1}\left|v\left(\rho r e^{i \theta}, \sigma s e^{i n}\right)\right| d \rho \\
& \leq c(\alpha, p)\left(\left\|v_{r, s}\left(\cdot, \sigma e^{i n}\right)\right\|_{p}+\left\|v_{r, s}\left(\cdot, \sigma e^{i \eta}\right)\right\|_{p}^{\alpha p} M\left(r e^{i \theta}, \sigma w\right)^{1-\alpha p}\right) .
\end{aligned}
$$

Integrating (3.5) with respect to $(1-\sigma)^{\alpha-1} d \sigma$, we get

$$
\text { (3.6) } \begin{aligned}
\left|I^{\alpha, \alpha} v(z, w)\right| & \leq C(\alpha, p)\left(\int_{0}^{1}(1-\sigma)^{\alpha-1}|| v_{r, s}\left(\cdot, \sigma e^{i n}\right) \|_{p} d \sigma\right. \\
& \left.+\int_{0}^{1} M\left(r e^{i \theta}, \sigma \omega\right)^{1-\alpha p}\left\|v_{r, s}\left(\cdot, \sigma e^{i n}\right)\right\|_{p}^{\alpha p}(1-\sigma)^{\alpha-1} d \sigma\right) .
\end{aligned}
$$

We take $q$ - means on both sides of (3.6) with respect to $\frac{d \theta}{2 \pi}$ and use Minkowski's inequalities in their discrete and continuous forms to get 
(3.7) $\quad\left(\int_{0}^{2 \pi}\left|I^{\alpha, \alpha} v\left(r e^{i \theta}, s e^{i \eta}\right)\right|^{q} \frac{d \theta}{2 \pi}\right)^{1 / q}$

$$
\begin{aligned}
& \leq C(\alpha, p)\left(\int_{0}^{1}(1-\sigma)^{\alpha-1}\left\|v_{r, s}\left(\cdot, \sigma e^{i \eta}\right)\right\|_{p} d \sigma\right. \\
& \left.\quad+\int_{0}^{1}\left(\int_{0}^{2 \pi} M\left(r e^{i \theta}, \sigma \omega\right)^{p} \frac{d \theta}{2 \pi}\right)^{\frac{1-\alpha p}{p}}\left|v_{r, s}\left(\cdot, \sigma e^{i \eta}\right)\right|_{p}^{\alpha p}(1-\sigma)^{\alpha-1} d \sigma\right) \\
& \leq C(\alpha, p) \int_{0}^{1}(1-\sigma)^{\alpha-1}|| v_{r, s}\left(\cdot, \sigma e^{i \eta}\right) \|_{p} d \sigma .
\end{aligned}
$$

We used the maximal theorem

$$
\int_{0}^{2} M\left(r e^{i \theta}, \sigma s e^{i \eta}\right) p \frac{d \theta}{2 \pi} \leq C(p)\left\|v_{r, s}\left(\cdot, \sigma e^{i \eta}\right)\right\|_{p}^{p},
$$

to get the last inequality in (3.7). Next, we set

$$
M(n)=\sup _{0 \leq \sigma<1}\left\|v_{r, s}\left(\cdot, \sigma e^{i \eta}\right)\right\|_{p}
$$

By Lerma 3.3 , we have

$$
\left\|v_{r, s}\left(\cdot, \sigma e^{i n}\right)\right\|_{p} \leq C(p)\left\|v_{r, s}\right\|_{p}(1-\sigma)^{-1 / p} .
$$

By (3.8) and (3.9), we have as before

$$
\begin{aligned}
& \int_{0}^{1}\left\|v_{r, \delta}\left(\cdot, \sigma e^{i n}\right)\right\|_{p}(1-\sigma)^{\alpha-1} d \sigma \\
& \leq C(p) \| v_{r, \delta}||_{p} \int_{0}^{\lambda}(1-\sigma)^{\alpha-\frac{1}{p}-1} d \sigma+M(\eta) \int_{\lambda}^{1}(1-\sigma)^{\alpha-1} d \sigma .
\end{aligned}
$$

We set $\lambda=0$ if $M(n) \leq\left\|v_{r, s}\right\|_{p}$ and $\lambda=1-\left(\frac{\left\|v_{r, s}\right\|_{p}}{M(n)}\right)^{p}$. otherwise. We have then for any $n$,

$$
\begin{aligned}
\int_{0}^{1}\left\|v_{r, \delta}\left(\cdot, \sigma e^{i n}\right)\right\|_{p}(1-\sigma)^{\alpha-1} d \sigma & \leq c(\alpha, p)\left(\left\|v_{r, s}\right\|_{p}\right. \\
& +\left\|v_{r, s}\right\|_{p}^{\alpha p} M(n)^{1-\alpha p)} .
\end{aligned}
$$


If we take $q$ - means on both sides with respect to $\frac{d n}{2 \pi}$, we have

$$
\begin{aligned}
& \left.\left(\int_{0}^{2 \pi} r \int_{0}^{1}\left\|v_{r, s}\left(\cdot, \sigma e^{i n}\right)\right\|_{p}(1-\sigma)^{\alpha-1} d \sigma\right)^{q} \frac{d \eta}{2 \pi}\right)^{1 / q} \\
& \leq c(\alpha, p)\left(\left\|v_{r, s}\right\|_{p}+\left\|v_{r, s}\right\| \|_{p}^{\alpha p}\left(\int_{0}^{2} M(n)^{p} d n\right)^{1 / q}\right) \\
& \leq c(\alpha, p)\left\|v_{r, s}\right\|_{p}
\end{aligned}
$$

by Lemma 3.4. If we note that $\log \frac{1}{Q} \sim 1-\rho$ as $\rho \rightarrow 1^{-}$and we combine $(3.7)$ and $(3.10)$, we have

$$
M_{q}\left(r, s ; I^{\alpha, \alpha} v\right) \leq C(\alpha, p)\left\|v_{r, s}\right\|_{p}
$$

So

$$
\left\|I^{\alpha, \alpha} v\right\|_{q} \leq c(\alpha, p)\|v\|_{p}
$$

\section{Proof of the main theorem}

By Proposition 2.2, $D^{\frac{1}{2}}, \frac{\frac{1}{2}}{2} v \in h^{2}\left(U^{2}\right)$. By Theorem 3.5, for any $\alpha$ $\left(0<a<\frac{1}{2}\right)$,

$$
I^{\alpha, \alpha} D^{\frac{1}{2}}, \frac{1}{2} v=D^{\frac{1}{2}-\alpha}, \frac{1}{2}-\alpha v \in h^{p}\left(U^{2}\right)
$$

where $p=\frac{2}{1-2 \alpha}$

Taking $\alpha$ arbitrarily close to $\frac{1}{2}$, we see that $v \in h^{p}\left(U^{2}\right)$ for any $p>0$. By Proposition 2.3, for each $p(1<p<\infty),|v|^{p}$ has a 2-harmonic majorant in $u^{2}$. If $p \leq 1$, then $|v|^{p} \leq|v|^{2}+1$. By the assertion above, $|v|^{2}+1$ has a 2-harmonic majorant; so does $|v|^{p}$. This completes the proof.

\section{References}

[1] P.L. Duren, Theory of $H^{P}$ spaces, (Academic Press, New York, NY. 1970).

[2] T.M. Flett, "The dual of an inequality of Hardy and Littlewood and some related inequalities", J. Math. Anal. Appl. 38 (1972), 746-765. 
[3] J.B. Garnett, Bounded analytic functions, (Academic Press, New York, NY 1981).

[4] G.H. Hardy and J.E. Littlewood, "Some properties of fractional integrals, II", Math. 2. 34 (1932), 403-439.

[5] G.H. Hardy and J.E. Littlewood, "Theorems concerning mean values of analytic or harmonic functions", Quart. J. Math. 12 (1941), 221-256.

[6] H.O. Kim, "Derivatives of Blaschke products", Pacific J. Math., 114 (1984), 175-189.

[7] H.O. Kim, "On a theorem of Hardy and Littlewood on the polydisc", Proc. Amer. Math. Soc. (to appear).

[8] W. Rudin, Function theory in polydiscs, (W.A. Benjamin, Inc., New York, NY, 1969).

[9] S. Yamashita, "Dirichlet-finite functions and harmonic majorants", IZZinois J. Math., 25 (1981), 626-631.

Department of Applied Mathematics,

Korea Advanced Institute of Science and Technology,

P.O. Box 150,

Cheongryang,

seoul,

Korea. 